\author{
N. Mandolesi \\ Istituto TESRE-CNR \\ Via de' Castagnoli, 1 \\ 40126 Bologna, Italy \\ D.J. Hegyi \\ Department of Physics \\ University of Michigan \\ Ann Arbor, Michigan USA
}

P. Crane

European Southern Observatory

Karl Schwarzschildstrasse, 2

D-8045 Garching, Western Germany

ABSTRACT. Observations of the interstellar lines of $\mathrm{CN}$ near $3875 \AA$
toward $\zeta$ Oph provide precise measurements of the Microwave Background
Radiation (MBR) temperature and determine the intrinsic 1inewidth
necessary for the saturation correction. We report $\mathrm{T}_{\mathrm{MBR}}=2.74 \pm 0.05 \mathrm{~K}$ at
$\lambda=2.64 \mathrm{~mm}$ and $\mathrm{T}_{\mathrm{MBR}}=2.75(+0.24,-0.29) \mathrm{K}$ at $\lambda=1.32 \mathrm{~mm}$.

\title{
INTRODUCTION
}

The $\mathrm{CN}$ absorption line technique for determining the temperature of the MBR can provide results with precision better than $2 \%$ at $\lambda=2.64 \mathrm{~mm}$. This can be compared to the best microwave radiometer measurements so far reported (Smoot et al., 1985; Mandolesi et a1., 1986) which have an accuracy of $5 \%$ in the millimeter region.

The present results were obtained in the summer of 1984 and 1985 at the ESO $1.5 \mathrm{~m}$ CAT coupled to a Coude' spectrograph. The data were recorded on a Reticon detector with a resolution of up to 156,000 .

The interstellar cloud in the direction of $\zeta$ Oph is particularly well suited to these observations because it is a single cloud of relatively large column density and has been well studied (Morton, 1975) in the past; thus many potential sources of error are known to be unimportant.

\section{DATA AND ANALYSIS}

of the 22 nigths assigned to this project, the weather allowed only seven nights data of sufficient quality for our purposes. These data were analysed in the standard way to remove instrumental effects and then wavelength calibrated. Non stochastic events limited our ability to reduce noise by adding data from several nights.

The 1984 observations were obtained with a resolution of 156,000 allowing us to determine the intrinsic width of the $\mathrm{CN}$ lines. The observed width of the $R(0)$ line was measured to be $31.8 \pm 0.3 \mathrm{~m} \AA$ (FWHM) 
Table I

Summary of CN Results at $\lambda=2.64 \mathrm{~mm}$.

\begin{tabular}{cccccc}
\hline \multirow{2}{*}{ Lines } & $\begin{array}{c}\text { Equivalent } \\
\text { Widths } \\
(\mathrm{m} \AA)\end{array}$ & $\mathrm{T}$ & $\Delta \mathrm{T}_{\text {sat }}$ & $\Delta \mathrm{T}_{\text {elec }}$ & $\mathrm{T}_{\mathrm{MBR}}$ \\
& & $(\mathrm{K})$ & $(\mathrm{K})$ & $(\mathrm{K})$ & $(\mathrm{K})$ \\
\hline
\end{tabular}

$\frac{R(1)}{R(0)} \quad \frac{2.420(51)}{7.646(91)} \quad 2.956(31) \quad-0.159(3) \quad-0.060$ (40) 2.737 (50)

$\frac{P(1)}{R(0)} \quad \frac{1.254(67)}{7.646(91)} \quad 3.015(71) \quad-0.197$ (3) -0.060 (40) 2.758 (82)

Table II

Summary of CN Results at $\lambda=1.32 \mathrm{~mm}$

\begin{tabular}{ccccc}
\hline \multirow{3}{*}{ Lines } & $\begin{array}{c}\text { Equivalent } \\
\text { Widths } \\
(\mathrm{m \AA})\end{array}$ & $\mathrm{T}$ & $\Delta \mathrm{T}_{\text {sat }}$ & $\mathrm{T}_{\mathrm{MBR}}$ \\
& & $(\mathrm{K})$ & $(\mathrm{K})$ & $(\mathrm{K})$ \\
\hline
\end{tabular}

$\frac{R(2)}{R(1)} \quad \frac{0.072(26)}{2.420(51)} \quad 2.78(+0.26 ;-0.30) \quad-0.03 \quad 2.75(+0.25 ;-0.29)$

$\frac{R(2)}{P(1)} \quad \frac{0.072(26)}{1.254(67)} \quad 2.75(+0.27 ;-0.31) \quad-0.01 \quad 2.74(+0.27 ;-0.30)$

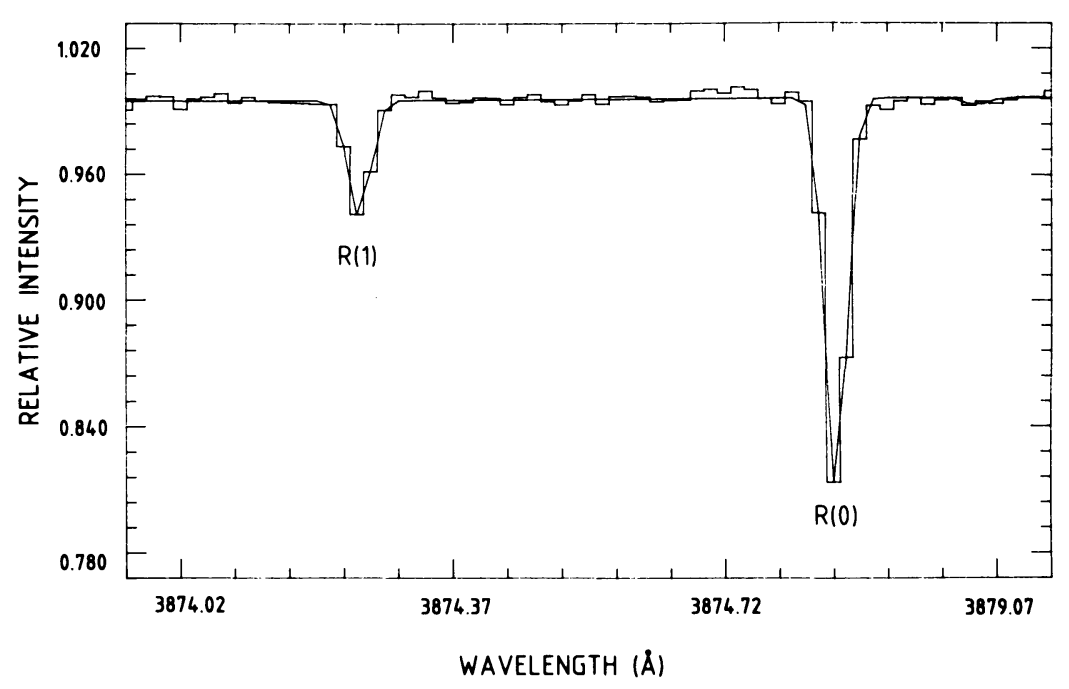

Figure 1: Plot of $C N R(0)$ and $R(1)$ lines. Solid line is a fit to the data. Wavelengths are observed not rest wavelengths. 
(Fig. 1), which is assumed to be the convolution of an intrinsic line profile with a FWHM of $19.0 \pm 0.5 \mathrm{~mA}$ and the instrumental profile of $24.8 \pm 0.4 \mathrm{m \AA}$.

The CN linewidth of $19.0 \mathrm{~m} \AA$ implies a velocity spread in the cloud of $0.88 \pm 0.02 \mathrm{~km} / \mathrm{s}$; this value should be compared with a value of $1.3 \mathrm{~km} / \mathrm{s}$ reported by Hegyi et al. (1972). For a number of reasons (Crane et al., 1986) we prefer our measured value. We note however that the choice of $1.3 \mathrm{~km} / \mathrm{s}$, in making saturation corrections, would increase our final $\mathrm{T}_{\mathrm{MBR}}$ at $2.64 \mathrm{~mm}$ by only $0.05 \mathrm{~K}$.

Ourr final equivalent widths are given in Table I and II together with errors. Also shown in the Tables are the $\mathrm{CN}$ excitation temperatures $\mathrm{T}$ at $\lambda=2.64$ and $1.32 \mathrm{~mm}$ before saturation corrections.

The Microwave Background Radiation temperature is derived from $\mathrm{T}$ ( Table $\mathrm{I}$ and $\mathrm{II}$ ) by making the saturation correction $\Delta \mathrm{T}$ and a correction for local electron excitation $\Delta \mathrm{T}$. (Meyer and Jurat, 1985). At $\lambda=1.32$ $\mathrm{mm}$ the correction for electron excitation is not necessary.

\section{CONCLUSIONS}

At $\lambda=2.64 \mathrm{~mm}$ the $\mathrm{CN}$ technique gives a result already accurate to $2 \%$. However this error is dominated by the uncertainty in the local excitation correction (Table I); hence further optical observations will not improve this result substantially.

At $\lambda=1.32 \mathrm{~mm}$ the precision in $\mathrm{T}_{\mathrm{MBR}}$ is dominated by the uncertainty in the $R(2)$ equivalent width. In this case more precise optical observations could improve the situation.

Our analysis has, so far, not considered one possible source of systematic error in the final $T_{M B R}$ values. We have assumed that the width of the $R(0)$ line is dominated by the velocity spread in a single cloud and not by the superposition of several clouds each with a low internal velocity spread. If this were the case then our saturation correction would be too small because the lines would be intrinsically narrower. We note that any effect of this sort would reduce the final $\mathrm{T}_{\text {MBR }}$ values.

However we have some confidence that our hypothesis is correct since our saturation corrected temperatures give very nearly the same values by using $R(1)$ and $P(1)$ lines.

\section{References}

Crane, P., Hegyi, D.J., Mandolesi, N., and Danks, A.C., 1986, Ap. J., 309.

Hegyi, D.J., Traub, W., and Carleton, N.P., 1972, Phys Rev. Letters, 28, 1541.

Mandolesi, N., Calzolari, P., Cortiglioni, S., Morigi, G., Danese, L., and De Zotti, G., 1986, Ap. J., 310.

Meyer, D.M., and Jura, M., 1985, Ap. J., 297, 119.

Morton, D.C., 1975, Ap. J., 197, 85.

Smoot, G.F., De Amici, G., Friedman, S.D., Witebsky, C., Sironi, G. Bonelli, G., Mandolesi, N., Cortiglioni, S., Morigi, G. Partridge, R.B., Danese, L., and De Zotti, G., 1985, ap. J. (Letters), 291, L23. 